\title{
Error Aware Distributed Space-Time Decoding for Regenerative Relay Networks
}

\author{
Chao Zhang \\ Department of Information and Communication Engineering, \\ Xi'an Jiaotong University, Xi'an, China \\ Email: chaozhang@mail.xjtu.edu.cn
}

\begin{abstract}
In this paper, we examine distributed space-time decoding structure for regenerative wireless relay networks. Given the demodulation error at the regenerative relays, we provide a general framework of error aware distributed spacetime decoding at the destination, where the receiver is assumed to know the demodulation error probability at relays. Considering the high computational complexity of optimal Maximum Likelihood (ML) decoder, we also propose two low-complexity decoding structures, Max-Log decoder and Max-Log-Sphere decoder. Simulations show that error aware distributed spacetime decoding can improve system performance greatly without high system overload, and Max-Log decoder and Max-LogSphere decoder can efficiently reduce decoding complexity with neglectable performance degradation.
\end{abstract}

\section{INTRODUCTION}

Relay-assisted communication is a promising strategy that exploits spatial diversity available among a collection of distributed single antenna terminals for both centralized and decentralized wireless networks. In most relay networks, a two-stage relaying strategy is used. In the first stage, a source transmits and all relays listen; in the second stage, the relays cooperate to forward the source symbols to the destination. Generally speaking, the relay functions can be separated into two types, regenerative and non-regenerative. If the relay processes the received signal and forwards the processed symbols, we call it regenerative relay, such as Decode-and-Forward (DCF) [1] and Demodulation-and-Forward (DMF) [2]. Otherwise, we call non-regenerative relay, such as Amplify-andForward (AF) [1]

It is well-known that the channel between source and relay is unreliable because of fading and noise. The relay receives an attenuated version of the source signal. AF relaying scheme amplifies noise. DCF scheme always using cyclic redundancy check $(\mathrm{CRC})$ will cause interruptions when the relay detects errors from the received message. DMF scheme is a trade-off between $\mathrm{AF}$ and $\mathrm{DCF}$ in relay processing. Relay can always keep a transmit link from the source, and detects and possibly decodes the source signal [4]. Moreover, the DCF scheme can also be considered as a special case of DMF if we consider the null signal as one choice of the modulation constellation. Therefore, in this paper, we treat DMF as the object to be studied for regenerative relay networks. However, DMF relay has an important disadvantage, which is the error produced in relay's Maximum Likelihood demodulation degrades the effective SNR at the destination significantly, which is called error propagation [5]. For distributed space-time coding system in regenerative relay networks, the degradation is more drastic [6],[7]. In [4], we proposed a threshold based scheme to minimize the error propagation, which is an active mechanism equipped in relays but subject to the large computation complexity.

In this paper, we intend to investigate the ML decoding structure where the destination is able to be aware of the error probability at the relays. The error probability at relay is a monotonic decreasing function of received SNR at relay, so the destination can estimate the error probability through training sequences which is transmitted by source and amplified by relay. Meanwhile, each relay also transmits its training sequence to estimate the relay-destination channel [6], [15]. Therefore, the error aware distributed space-time decoding is rational. Through analyzing the error aware ML detecting, we give a general framework of error aware distributed spacetime decoding at the destination. Because the ML decoder is composed of multiple likelihood function generators, the computational complexity is too large to be affordable. Due to max-log approximation, we provide an Max-Log decoder based on Csiszár-Tusnady algorithm [12]. Moreover, to reduce the complexity further, we propose a Max-Log-Sphere decoder which is also based on max-log approximation. Also, we provide complexity comparisons of these decoders in terms of elementary operation number. Finally, simulations verify the low complexity performances.

\section{System Model}

We consider a wireless network with $N$ randomly placed relay nodes, relay $i=1, \ldots, N$, one source node $S$, and a destination node $D$. Each node is equipped with only a single antenna and uses the Half-duplex mode. Denote the channel from the source to the $i$ th relay as $f_{i}$ and the channel from the $i$ th relay to the destination as $h_{i}$. Assume that $f_{i}$ and $h_{i}$ are independent complex Gaussian random variables with zeromean and variance $\delta_{s i}^{2}$ and $\delta_{i d}^{2}$, respectively. Receiving noise is assumed as complex Gaussian random variable with zeromean and unit-variance. We assume a block fading channel model, where channel gain stays constant during a time block and changes from block to block. We also assume that the instantaneous channel is unknown to the transmitting 
node but perfectly known at receiving node. Assume that the source wishes to send the signal $\mathbf{s}=\left[s_{1}, s_{2}, \ldots, s_{T}\right]^{T}$ to the destination, where $s_{i} \in \mathcal{A}$, and $\mathcal{A}$ is a finite constellation with average power $1 / T$ and $T$ is the length of each time slot. Hence, $E\left\{\mathbf{s}^{H} \mathbf{s}\right\}=1$. Assume $\mathbf{s}$ is in the codebook $\mathcal{S}=\left\{\mathbf{s}_{0}, \ldots, \mathbf{s}_{L-1}\right\}$, where $L$ is the cardinality of the codebook. For convenience, we consider all transmit power is unit.

During the first stage, source transmits $\mathbf{s}_{l}, l \in[0, L-1]$ to all relays, then each relay tries to demodulate the received signal. At the $i$ th relay, denote the received symbol vector out of demodulator is $\hat{\mathbf{s}}_{i}$, and $\hat{\mathbf{s}}_{i} \in \mathcal{S}$. The demodulation probability is given by

$P\left(\hat{\mathbf{s}}_{i} \mid f_{i}, \mathbf{s}_{l}\right)= \begin{cases}\mathcal{Q}\left(\sqrt{\frac{1}{2}\left|f_{i}\right|^{2}\left|\mathbf{s}_{l}-\mathbf{s}_{i}\right|^{2}}\right), \quad \hat{\mathbf{s}}_{i} \neq \mathbf{s}_{l} \\ 1-\sum_{j=1}^{L-1} \mathcal{Q}\left(\sqrt{\frac{1}{2}\left|f_{i}\right|^{2}\left|\mathbf{s}_{l}-\mathbf{s}_{i}\right|^{2}}\right), \quad \hat{\mathbf{s}}_{i}=\mathbf{s}_{l}\end{cases}$

where $\mathcal{Q}(x)=\frac{1}{\sqrt{2 \pi}} \int_{x}^{\infty} e^{-\frac{t^{2}}{2}} d t$. At the $i$ th relay, the received signal $\hat{\mathbf{s}}_{i}$ is mapped onto a $T \times 1$ vector (not necessary), $\mathcal{F}_{i}\left(\hat{\mathbf{s}}_{i}\right)$, as processed at one antenna of co-located space-time coding transmitter. We assume the map function $\mathcal{F}_{i}$ is invertible. Therefore, there are $L$ possible transmitted vectors to destination for the $i$ th relay, because $\hat{\mathbf{s}}_{i}$ could be any vector in $\mathcal{S}$. Herein, we assume all mapping functions $\mathcal{F}_{i}, i=1, \ldots, N$ are different with each other but it is not necessary. Then, all relays transmit the mapped vectors to the destination. At the destination, the received signal is

$$
\mathbf{Y}=\left[\mathcal{F}_{1}\left(\hat{\mathbf{s}}_{1}\right), \ldots, \mathcal{F}_{N}\left(\hat{\mathbf{s}}_{N}\right)\right] \mathbf{H}+\mathbf{N}
$$

where $\mathbf{Y}=\left[y_{1}, \ldots, y_{T}\right]^{T}$ is the received signal, $\mathbf{H}=$ $\left[h_{1}, \ldots, h_{N}\right]^{T}$ is the relay to destination channel vector and $\mathbf{N}$ is Gaussian white noise. Define a codebook $\mathcal{C}=$ $\left\{\left[\mathcal{F}_{1}\left(\hat{\mathbf{s}}_{1}\right), \ldots, \mathcal{F}_{N}\left(\hat{\mathbf{s}}_{N}\right)\right]\right\}$. Clearly, $\mathcal{C}$ includes $L^{N}$ elements. We define the $k$ th element of $\mathcal{C}$ is $\mathbf{C}_{k}=\left[\mathbf{c}_{1 k}, \ldots, \mathbf{c}_{N k}\right]$, where $\mathbf{c}_{i k} \in\left\{\mathcal{F}_{i}\left(\hat{\mathbf{s}}_{i}\right)\right\}$. Thus, if $\mathbf{C}_{k}$ is transmitted, we can express (2) as

$$
\mathbf{Y}=\mathbf{C}_{k} \mathbf{H}+\mathbf{N}
$$

Denote the inverse function of $\mathcal{F}$ as $\mathcal{F}^{-1}$. Then, we have

$$
P\left(\mathbf{C}_{k} \mid \mathbf{F}, \mathbf{s}_{l}\right)=\prod_{i=1}^{N} P\left(\mathcal{F}_{i}^{-1}\left(\mathbf{c}_{i k}\right) \mid f_{i}, \mathbf{s}_{l}\right)
$$

where $\mathbf{F}=\left[f_{1}, \ldots, f_{N}\right]^{T}$. So, by (1) we can derive the exact value of (4). For $\mathbf{C}_{k}$, traditional ML decoder can be used. So, there is

$$
P\left(\mathbf{Y} \mid \mathbf{H}, \mathbf{C}_{k}\right)=\frac{1}{\pi^{N}} \exp \left(-\left(\mathbf{Y}-\mathbf{C}_{k} \mathbf{H}\right)^{\mathcal{H}}\left(\mathbf{Y}-\mathbf{C}_{k} \mathbf{H}\right)\right)
$$

\section{ERror AWARE MAXimum LiKelihood DeCODER}

In this section, we provide a general Maximum Likelihood decoder for distributed space-time codes in regenerative relay networks. First of all, destination should know the channel information in this relay networks. The channel from relays to the destination $h_{i}, \quad i=1, \ldots, N$ can be estimated through pilot symbols which are transmitted by each relay before data

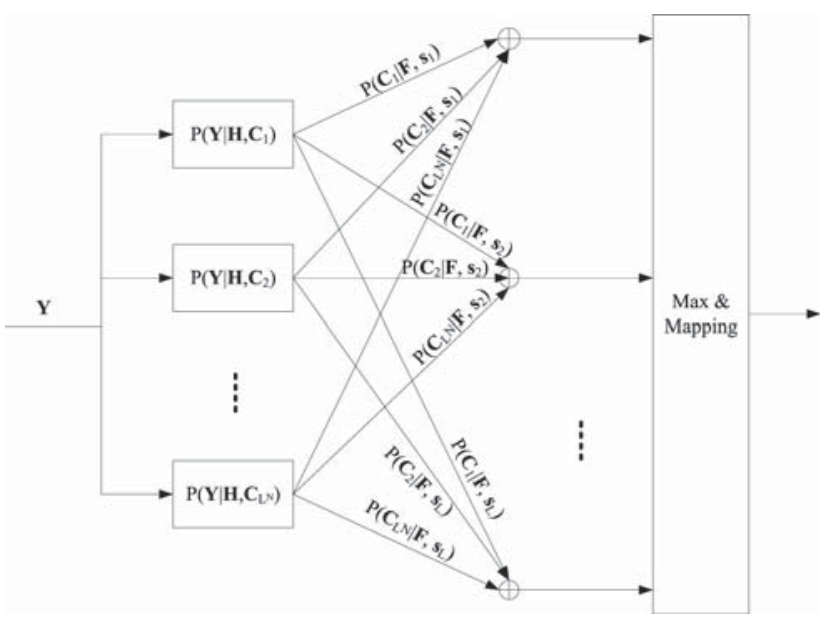

Fig. 1. Error Aware ML decoder

transmission. Here, we assume all the estimators are ideally accurate without any error. To let the destination know the demodulation error happening at each relay, we propose the following scheme. The source transmits pilot symbols to all relays through channels $f_{1}, \ldots, f_{N}$. Without demodulating, each relay maps the noise version signal to a vector like the scheme proposed in [9]. Then, relay transmits the vector to the destination like the Amplify-and-Forward based distributed space-time coding [10]. Therefore, the information about the channel between source and destination carried by amplified pilots, i.e., $f_{i} h_{i}, \quad i=1, \ldots, N$, can be estimated at the destination. Knowing $h_{i}$ at the destination, then $f_{i}$ also can be estimated. Because the signal vector set $\mathcal{S}$ and all mapping functions $\left\{\mathcal{F}_{i}\right\}$ are known at the destination as a prior knowledge, (4) can be derived at the destination. Even though this is not a perfect estimator in practice, our assumptions allows us to focus only on the performance of error aware DSTC. The degradation of performance due to channel estimation do not come under the scope of this paper.

If the transmitted signal is $\mathbf{s}_{l}$, the likelihood function is

$$
P\left(\mathbf{Y} \mid \mathbf{F}, \mathbf{H}, \mathbf{s}_{l}\right)=\sum_{k=1}^{L^{N}} P\left(\mathbf{Y} \mid \mathbf{H}, \mathbf{C}_{k}\right) P\left(\mathbf{C}_{k} \mid \mathbf{F}, \mathbf{s}_{l}\right)
$$

Therefore, the Error Aware ML decoder is

$$
\arg \max _{1 \leq l \leq L}\left\{P\left(\mathbf{Y} \mid \mathbf{F}, \mathbf{H}, \mathbf{s}_{l}\right)\right\}
$$

Utilize (4)-(6), then (7) is derived. By (5), $P\left(\mathbf{Y} \mid \mathbf{H}, \mathbf{C}_{k}\right)$ is independent of $\mathbf{s}_{l}$, so that according to the estimated channel $\mathbf{H}, P\left(\mathbf{Y} \mid \mathbf{H}, \mathbf{C}_{k}\right)$ can be calculated first. Then, using the amplified pilot, $P\left(\mathbf{C}_{k} \mid \mathbf{F}, \mathbf{s}_{l}\right)$ are also derived. Therefore, the ML decoder can be built in Fig. 1, where we show the structure of error aware ML decoder for regenerative distributed spacetime coding. Note that there are $L$ adders and $L^{N}$ likelihood function generators.

The complexity of the Error aware ML decoder equals to that of $L^{N}$ co-located space-time decoders, it is too large to be affordable if the signal block length, modulation order and 
the number of relay are considerable large. [2] considered a piecewise-linear approximation to solve a similar problem, but in this case it is also too complicated to design an approximation function.

\section{LOW-COMPLEXITY ERROR AWARE DECODERS}

In this section, we will introduce two low-complexity error aware decoders through analyzing and simplifying the structure of ML decoder. Without subject to a specific decoding function, we focus on fully exploiting the general structure of ML decoder. First, we use Max-Log approximation to derive a Max-Log error aware decoder which can work with CsiszárTusnady algorithm. Second, to reduce the complexity further, sphere decoding also is combined into the Max-Log decoder, which is called Max-Log-Sphere decoder.

\section{A. Error Aware Max-Log Decoder}

Substitute (6) into (7), there is

$$
\arg \max _{1 \leq l \leq L}\left\{\sum_{k=1}^{L^{N}} P\left(\mathbf{Y} \mid \mathbf{H}, \mathbf{C}_{k}\right) P\left(\mathbf{C}_{k} \mid \mathbf{F}, \mathbf{s}_{l}\right)\right\}
$$

Because $\log (x)$ is an increasing monotonic function, the ML decoder can be rewritten as

$$
\arg \max _{1 \leq l \leq L}\left\{\log \left(\sum_{k=1}^{L^{N}} \exp \left\{-\frac{1}{2}\left\|\mathbf{Y}-\mathbf{C}_{k} \mathbf{H}\right\|^{2}+\lambda_{k, l}\right\}\right)\right\}
$$

According to (5) and max-log approximation in [11], we derive

$$
\arg \max _{1 \leq l \leq L}\left\{\max _{1 \leq k \leq L^{N}}\left\{-\left\|\mathbf{Y}-\mathbf{C}_{k} \mathbf{H}\right\|^{2}+\lambda_{k, l}\right\}\right\}
$$

where $\lambda_{k, l}=\log \left(P\left(\mathbf{C}_{k} \mid \mathbf{F}, \mathbf{s}_{l}\right)\right)$.

We can see that decoding distributed space-time code becomes searching a two-dimension array, which is indexed by $(k, l)$. Intuitively, this decoder also needs $L^{N}$ ML detector like ML decoder. The only difference is that calculating the likelihood function of each symbol vector does not need crosscomputation. However, double maximization problem can take advantage of Csiszár-Tusnady algorithm to reduce computing [12]. Because the set $\left\{\left\|\mathbf{Y}-\mathbf{C}_{k} \mathbf{H}\right\|^{2}\right\}$ is a set of distance measure which is one-one mapped to a probability distribution set and $\left\{\lambda_{k, l}\right\}$ is the set of probability distribution, moreover, $\left\{\lambda_{k, l}\right\} \leq 0$, then (10) can be seemed to seek the vector who has the minimum sum distance which equals to the distance from $\mathbf{s}_{l}$ to $\mathbf{C}_{k}$ plus the distance from $\mathbf{C}_{k}$ to $\mathbf{Y}$. Thus, Csiszár-Tusnady algorithm does converge to the maximum element [12]. We summarize the iterative Max-Log decoder as following (Fig. 2).

1) Initialization: Set error probability set $\mathcal{P}$. Take any element of $\mathbf{C}_{k} \in \mathcal{C}$ and compute $\mathbf{D}_{k}=-\left\|\mathbf{Y}-\mathbf{C}_{k} \mathbf{H}\right\|^{2}$.

2) Step 1: Find the $l$ that makes $\mathbf{D}_{k}+\lambda_{k, l}$ is maximum for the chosen $k$, where $\lambda_{k, l} \in \mathcal{P}$ and is calculated by (4).

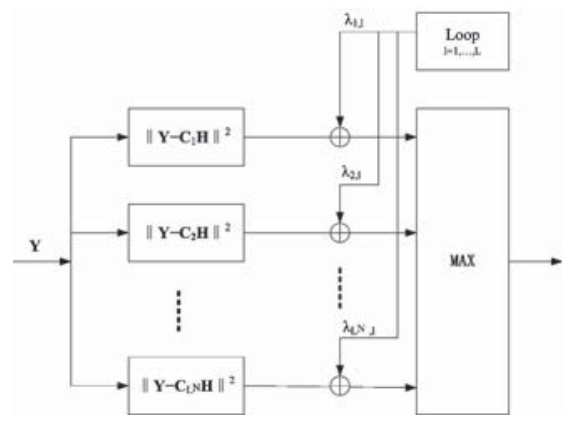

Fig. 2. Max-Log decoder

3) Step 2: Fix $l$ and find a $\mathbf{D}_{n} \in \mathcal{C}$ which makes $\mathbf{D}_{n}+\lambda_{n, l}$ is maximum.

4) Decision: If $n=k$, goto End, otherwise, goto Step 1 .

5) End: $\mathbf{s}_{l}$ is the decoded vector.

\section{B. Error Aware Max-Log Sphere Decoder}

If the length of vector $\mathbf{s}$ and the constellation size are sufficiently large, Max-Log decoder is also subject to the implementation. The largest computation is required for searching code set $\mathcal{C}$ with cardinality $L^{N}$. Reducing the decoder complexity depends on searching $\mathcal{C}$.

To state the Max-Log Sphere decoder, we first find the realvalued equivalent of (3), Define

$$
\begin{aligned}
& \tilde{\mathbf{Y}}=\left[\mathcal{R}\{\mathbf{Y}\}^{T}, \mathcal{I}\{\mathbf{Y}\}^{T}\right]_{2 T \times 1}^{T} \quad \tilde{\mathbf{H}}=\left[\mathcal{R}\{\mathbf{H}\}^{T}, \mathcal{I}\{\mathbf{H}\}^{T}\right]_{2 N \times 1}^{T} \\
& \tilde{\mathbf{N}}=\left[\mathcal{R}\{\mathbf{N}\}^{T}, \mathcal{I}\{\mathbf{N}\}^{T}\right]_{2 T \times 1}^{T} \tilde{\mathbf{C}}_{k}=\left[\begin{array}{cc}
\mathcal{R}\left\{\mathbf{C}_{k}\right\} & \mathcal{I}\left\{\mathbf{C}_{k}\right\} \\
-\mathcal{I}\left\{\mathbf{C}_{k}\right\} & \mathcal{R}\left\{\mathbf{C}_{k}\right\}
\end{array}\right]_{2 T \times 2 N}
\end{aligned}
$$

where $\mathcal{R}\{$.$\} and \mathcal{I}\{$.$\} denote real part and imaginary part.$ By (10), we yield

$$
\arg \min _{1 \leq l \leq L}\left\{\min _{1 \leq k \leq L^{N}}\left\{\left\|\tilde{\mathbf{Y}}-\tilde{\mathbf{C}}_{k} \tilde{\mathbf{H}}\right\|^{2}-\lambda_{k, l}\right\}\right\}
$$

For a specific $\mathbf{s}_{l}$, the decoding object is

$$
\begin{aligned}
& \arg \min _{1 \leq k \leq L^{N}}\left\{\left\|\tilde{\mathbf{Y}}-\tilde{\mathbf{C}}_{k} \tilde{\mathbf{H}}\right\|^{2}-\lambda_{k, l}\right\}= \\
& \arg \min _{1 \leq k \leq L^{N}}\left\{\left\|\tilde{\mathbf{Y}}-\left(\tilde{\mathbf{H}}^{T} \otimes \mathbf{I}\right) \operatorname{Vec}\left\{\tilde{\mathbf{C}}_{k}\right\}\right\|^{2}-\lambda_{k, l}\right\}
\end{aligned}
$$

where $\otimes$ is the Kronecker product operation. Obviously, we can use Sphere decoding method [13]-[14] to searching $\mathbf{C}_{k}$, which minimizes (12). Note that $\|\tilde{\mathbf{N}}\|^{2}=\|\tilde{\mathbf{Y}}-\tilde{\mathbf{C}} \tilde{\mathbf{H}}\|^{2}$ is a $\chi^{2}$ random variable with $2 N$ degrees of freedom. We choose the radius $r$ to be a linear function of the variance of $\|\tilde{\mathbf{N}}\|^{2}$

$$
r^{2}=2 \alpha N
$$

where the coefficient $\alpha$ is chosen in such a way that with a high probability $P_{f p}$ we can find a lattice inside a sphere

$$
\int_{0}^{2 \alpha N} \frac{x^{N-1}}{\Gamma(N)} e^{-x} d x
$$


where $\Gamma(N)=\int_{0}^{\infty} t^{N} e^{-t} d t$. Note that the radius is chosen based on the noise not on channel efficiency. As stated in [13], this point has a beneficial effect on the computational complexity.

For expression convenience, we define $\tilde{\mathbf{H}}^{T} \otimes \mathbf{I}=\mathbf{B}$ and $\operatorname{Vec}\left\{\tilde{\mathbf{C}}_{k}\right\}=\mathbf{X}$ with size $4 N T \times 1$. Therefore, searching $\mathbf{X}$ is equal to searching $\mathbf{C}_{k}$. Applying the idea of the Fincke Pohst algorithm, we search for the point $\mathbf{X}$ that belong to the geometric body described by

$$
r_{k, l}^{2} \geq(\mathbf{X}-\hat{\mathbf{X}})^{H} \mathbf{U}^{H} \mathbf{U}(\mathbf{X}-\hat{\mathbf{X}})-\sum_{i=1}^{N} \log \left(P\left(\mathbf{c}_{i k} \mid f_{i}, \mathbf{s}_{l}\right)\right)
$$

where $\hat{\mathbf{X}}=\mathbf{B}^{\dagger} \mathbf{Y}=\left(\mathbf{B}^{T} \mathbf{B}\right)^{-1} \mathbf{A}^{T} \mathbf{Y}$ and $\mathbf{U}$ is the low triangular matrix obtained from $Q R$ factorization of $\mathbf{B}$. The search radius $\dot{r}_{k, l}$ is chosen according to the statistical properties of noise and the decoding error at relays. Denote $4 N T=M$, then, a necessary condition for $x_{M}$, the $M$ th element of $\mathbf{X}_{k}$, is

$$
b_{M, M}^{2}\left(x_{M}-\hat{x}_{M}\right)^{2}-\delta_{k, l, M} \leq \dot{r}_{k, l}^{2}
$$

where $\delta_{k, l, M}$ is defined as

$$
\delta_{k, l, M}=\frac{1}{2 N} \log P\left(\mathbf{C}_{j, k} \mid f_{j}, \mathbf{s}_{l}\right), \quad j=\frac{1}{2} \bmod (M, 2 T)
$$

. Herein we allocate the additional weight $\delta_{k, l, m}$ averagely over $\mathrm{N}$ relays. Moreover, we define

$$
\dot{r}_{k, l, M-1}^{2}=\dot{r}_{k, l}^{2}-b_{M, M}^{2}\left(x_{M}-\hat{x}_{M}\right)^{2}+\delta_{k, l, M}
$$

and a new necessary condition can be written as

$$
\begin{aligned}
& b_{M-1, M-1}^{2}\left(x_{M-1}-\hat{x}_{M-1}+\frac{b_{M-1, M}}{b_{M-1, M-1}}\left(x_{M}-\hat{x}_{M}\right)\right)^{2}- \\
& \delta_{k, l, M-1} \leq \dot{r}_{k, l, M-1}^{2}
\end{aligned}
$$

In a similar fashion, one proceeds for $x_{M-2}$, and son on, and until all components of vector $\mathbf{X}$ are found. Note that the dominant difference between Max-Log-Sphere decoder and Sphere decoder proposed in [13] is that radius varies according to all possible codewords. In this Max-Log-Sphere decoder, for each $k$, we just check $\mathbf{X}_{k}$ whether to meet its radius. If there exists more than one $\mathbf{X}_{k}$ can meet the constraint (16) for $x_{m}$, keep these survival codeword and go to next codeword. If some of there codewords can not meet the new constraint, then drop them. That is to say for each lattice we must try all possible radiuses. For a specific $\mathbf{s}_{l}$, Max-Log-Sphere decoder can be summarized as follows.

Input $\mathbf{B}, \mathbf{Y}, \hat{\mathbf{X}}, r, \lambda_{k, l}, d$, Set $k=1$.

1) Set $\mathrm{m}=\mathrm{M}, \dot{r}_{k, l, M}^{2}=r^{2}-\|\tilde{\mathbf{Y}}\|^{2}+\|\mathbf{B} \hat{\mathbf{X}}\|, \hat{x}_{M \mid M+1}=\hat{x}_{M}$.

2) (Set bounds for $x_{m}$ ) Set $z_{k}=\frac{r_{k, l, m}}{b_{m, m}}$, $U B\left(x_{m}\right)=\left\lfloor z_{k}+\hat{x}_{m \mid m+1}\right\rfloor, x_{m}=\left\lceil-z_{k}+\hat{x}_{m \mid m+1}\right\rceil-d$.

3) (Check $\left.x_{m}\right)$ Set $x_{m}=\mathbf{X}_{k}(m)$, if $b_{m, m}^{2}\left(x_{m}-\hat{x}_{m \mid m+1}\right)^{2}>\dot{r}_{k, l, m}^{2}+\delta_{k, l, m}$ and $x_{m} \leq$ $U B\left(x_{m}\right)$, go to 5$)$, else to 4$)$.

4) (Increase $k$ ) $k=k+1$, If $k=L^{N}+1$, terminate algorithm, else go to 1 ).

\begin{tabular}{c|c}
\hline \hline & Average operation number \\
\hline ML decoder & $L^{N}[8 T N+2 L-1]-L$ \\
\hline Max-Log decoder & $L^{N}[8 T N-1]+L$ \\
\hline Max-Log-Sphere decoder & Eqa.(22) \\
\hline \hline
\end{tabular}

TABLE I

ELEMENTARY OPERATION NUMBER OF THREE DECODERS

5) (Decrease $m$ ) If $m=1$ go to 6). Else $m=m-$ $1, \hat{x}_{m, m-1}=\hat{x}_{m}+\sum_{j=m+1}^{M}\left(b_{k . j} / b_{m, m}\right)\left(x_{j}-\hat{x}_{j}\right)$, $\dot{r}_{k, l, m}^{2}=\dot{r}_{k, l, m+1}^{2}-r_{m+1, m+1}^{2}\left(x_{m+1}-\hat{x}_{m+1 \mid m+2}\right)^{2}+$ $\delta_{k, l, m+1}$, and go to 2 )

6) Solution found for $k$. Save $k, \mathbf{X}_{k}$ and exact distance $d_{k, l}$. and set $k=k+1$, if $k=L^{N}+1$, terminate algorithm, else go to 1 ).

After terminating the decoder algorithm for $\mathbf{s}_{l}$, select the $\mathbf{C}_{k}$ which achieves the minimum distance to $\tilde{\mathbf{Y}}$. Then through $L$ Max-Log-Sphere decoder with $l=1, \ldots, L$, choose the $\mathbf{s}_{l}$ which minimizes the distance to $\mathbf{Y}$.

Note that Max-Log-Sphere decoder needs estimating the noise variance of the receiver. However, Max-Log decoder using Eulerian distance and error probability is more realizable. Hence, there is a tradeoff between computational complexity and implementation to choose which one is suitable.

\section{Computational Complexity}

In this section, we analyze and compare the computational complexity of above three decoders. We use the average numbers of real elementary operation, $C_{p}$, (including addition, subtraction, multiplication and division) as a measure for computational complexity. We draw a table to show the computational complexity. Max-Log decoder mentioned herein is the iterative Max-log decoder. The complexity of ML decoder and Max-Log decoder are easy to be done, we directly list them in Table I. Max-Log-Sphere decoder for a $\mathbf{s}_{l}$ has $L^{N}$ radiuses but each radius is only assigned for searching one possible $\mathbf{C}_{k}$. An arbitrary lattice point $\mathbf{X}_{k}$ belongs to a $m$ dimensional sphere of radius $r_{k, l}$ around the transmitted point $\mathbf{X}_{t}$ is given by the following incomplete Gamma function [13][14]

$$
P_{k, l}=\gamma\left(\frac{2 \alpha N+\lambda_{k, l}}{2\left(1+\operatorname{Pr}\left\|\mathbf{X}_{t}^{m}-\mathbf{X}_{k}^{m}\right\|^{2}\right)}, \frac{2 N-2 T+m}{2}\right)
$$

where $\mathbf{X}^{m}=\left[x_{M-m}, x_{M-m+1}, \ldots, x_{M}\right]^{T}$ and $\operatorname{Pr}$ is the relay transmit power, which is assumed as unit in above context. The number of elementary operation that the Max-Log-Sphere decoder performs per each visited point in dimension $m$ are

$$
C_{p}(k, l, m)=2 m+12
$$

Therefore, $C_{p}$ of Max-Log-Sphere decoder is yielded as

$$
C_{p}=\sum_{l=1}^{L} \sum_{k=1}^{L^{N}} \sum_{m=1}^{M} C_{p}(k, l, m) P_{k, l}
$$

Obviously, Max-Log decoder has a lower complexity than ML 
decoder. It is difficulty to compare Max-Log Sphere decoder with others, but in next section we will show simulation results to illustrate the differences.

\section{Simulation Results}

In this section, we provide the simulation results to show the proposed error aware decoders. We denote the total power noise ratio as the system signal noise ration (SNR) indicator. And half of total power is assigned for source transmit power and another half is equally divided by all relays. In this simulation, we adopt distributed linear dispersion code proposed in [10] as the coding scheme for its simplicity, where $\mathcal{F}_{i}(\mathbf{s})=\mathbf{A}_{i} \mathbf{s}$ and $\mathbf{A}_{i}$ is a random unitary matrix. For MaxLog-Sphere decoder, herein we set $P_{f p}=0.99$. All other parameters are the same with system model. We also should claim the non-error aware decoder is

$$
\arg \min _{\mathbf{s}}\|\mathbf{Y}-\mathbf{C}(\mathbf{s}) \mathbf{H}\|^{2}
$$

where $\mathbf{C}(\mathbf{s})=\left[\mathbf{A}_{1} \mathbf{s}, \mathbf{A}_{2} \mathbf{s}, \ldots, \mathbf{A}_{N} \mathbf{s}\right]$.

Fig. 4 demonstrates bit error rate (BER) performances of different decoders where two relays are employed and the signal modulation is BPSK. That is to say $T=N=2$. We can see that at high SNR regime error aware decoders achieve almost $5 \mathrm{~dB}$ gain than non-error aware decoder and outperform AF based DSTC scheme about $3 \mathrm{~dB}$. Thus it is worthy to bring slight system overhead for delivering channel estimation to improve the system performance. Over all SNR range, Max-Log decoder and Max-Log-Sphere decoder have nearly the same performance with ML decoder. Therefore, the degradation of max-log approximation is negligible. By carefully observing, we found the slope of BER curve decreases. Because the hard-decision error at relay limits the systems performance even though SNR is enough high.

In Fig. 5, we also simulate a 4 relay network to show the BER performance of that decoders. Herein, $T=N=4$ and modulation is QPSK. Similarly, error aware decoders can bring about $7 \mathrm{~dB}$ power gain than non-error aware decoder at 22 dB SNR. We can see that it is different from Fig. 4 that error aware decoders only achieve about $1.5 \mathrm{~dB}$ gain than $\mathrm{AF}$ based DSTC. The reason is that high-order modulation incurs more error after decoding at relays and enlarges error-propagation so that deceases the possible gain of error aware decoder. And in this case, the differences of three error aware decoders are more slight. It is interesting that the slop of BER curve does not decrease here. That is because more relays bring more error conditions and consume more power. Therefore, the slop decreasing threshold is larger than 2 relay with BPSK system. From both two figures, we can assert that error aware decoders can improve the system performance efficiently with little system cost.

For DSTC relay networks, [10] had proven that the maximum achievable diversity order is $\min \{N, T\}$. [16] addressed that demodulate-and-forward scheme in a relay network where direct link is available can only achieve half of maximum diversity. In our simulations, non-error aware DSTC has a even less diversity, i.e., the diversity of non-error aware DSTC

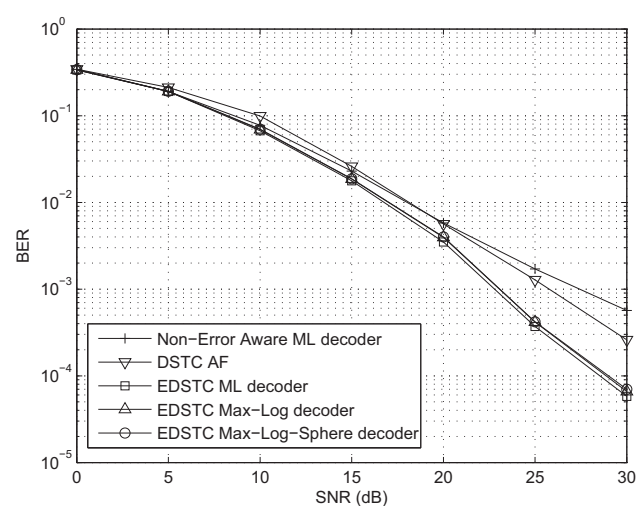

Fig. 3. BER performance of Error Aware decoders with 2 relays, BPSK

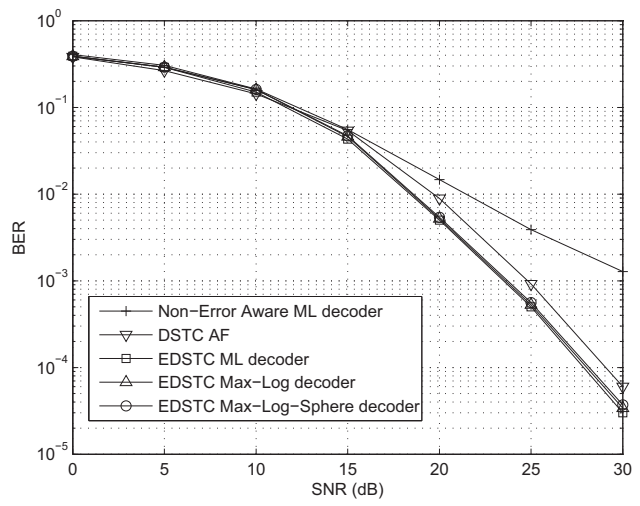

Fig. 4. BER performance of Error Aware decoders with 4 relays, QPSK

in Fig. 4, is 1 and in Fig. 5, is only 1.2. That is because there is no direct link in our model and direct link which does not produce demodulation error. Adding a direct link can increase the system diversity by one but adding on one relay could not gain advantages, but even get worse. Essentially speaking, demodulation error limits the diversity growing. On the other hand, EDSTC has a larger diversity than non-error aware DSTC. That is to say EDSTC gains advantages from the available error probabilities. Since there are also demodulation errors at relays, EDSTC can not achieve the full diversity.

Now, we will show the computational complexity of three error aware decoders by elementary operation number. Note that the operation number of Max-Log-Sphere decoder varies with unitary matrices $\mathbf{A}_{i}$ and the channel realization because of (12). We average the elementary operation number over 1000 channel realizations.

In Fig. 6, we show the average operation number of these three decoders when 2 relays are employed. Obviously, $C_{p}$ s of ML decoder and Max-Log decoder are independent of SNR. Max-Log decoder has a lower complexity than ML decoder. Max-Log-Sphere decoder needs less than half of operation numbers of ML decoder or Max-Log decoder. Of course, for 2 relay network, the operation number of ML decoder is trivial 


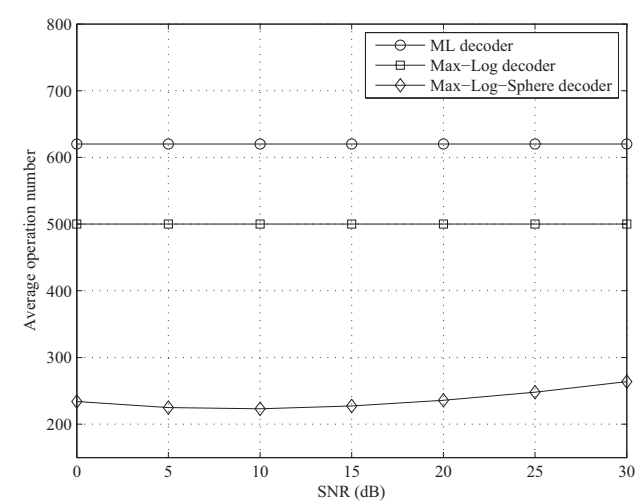

Fig. 5. Average operation number of three decoders with 2 relay, BPSK

compared with current hardware computing rate. However, for 4 relays with QPSK modulation scheme, it is too large to be affordable. Fig. 7 gives the elementary operation number in this case. We can see that ML decoder has $2.7445 \mathrm{e}+012$ operations! The operation number of Max-Log decoder is nearly $10 \%$ of that of ML decoder. It is notable that MaxLog-Sphere decoder needs only $0.1 \%$ operation number of ML decoder. Therefore, Max-Log-Sphere decoder achieves the same BER performance with optimal ML decoder but costs drastically low computation. Although Max-Log-Sphere has an attractive performance, the noise variance should be estimated first to calculate searching radius [13]. Max-Log decoder just utilizes Eulerian distance and error probabilities, therefore, it is a good trade-off for decoding structure between implementation and computational complexity. We can choose one of them due to different receivers.

\section{CONCLUSION}

In this paper, we provide a general framework of error aware distributed space-time decoder for regenerative relay networks. Through two-stage pilot symbols, the destination can estimate not only the relay-destination channel but also the error probability happening at relays. Using these estimated error, Maximum Likelihood decoder is provided. To reduce computational complexity, Max-Log decoder and Max-LogSphere decoder are also proposed by max-log approximation. Simulations show that error aware decoders can improve the performance drastically. Max-Log-Sphere decoder can achieve the same performance with ML decoder and needs far lower computational complexity. Without noise estimating, Max-Log decoder can make a good trade-off between implementation and computational complexity.

\section{ACKNOWLEDGMENT}

This work was supported by New Faculty Support Program of Xi' an Jiaotong University and was supported in part by the National Natural Science Foundation of China under Grant No. 60832007 and the National Hi-Tech Research and Development Plan of China under Grant No. 2009AA011801.

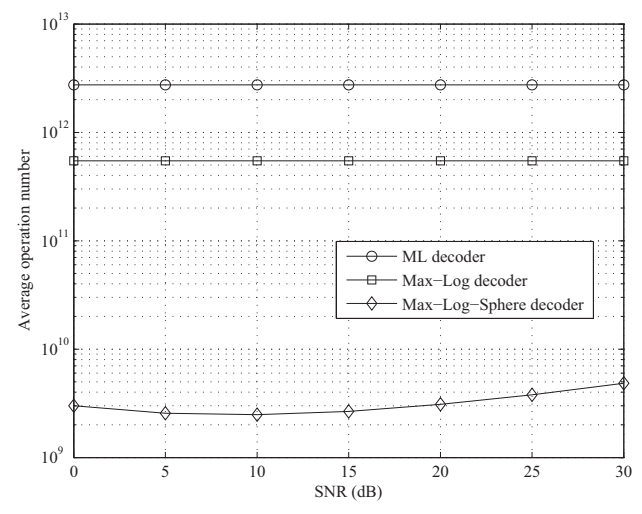

Fig. 6. Average operation number of three decoders with 4 relay, QPSK

\section{REFERENCES}

[1] J. Laneman, D. Tse, and G. Wornell, "Cooperative diversity in wireless networks: efficient protocols and outage behavior,'IEEE Trans. on Inform. Theory, vol. 50, no. 12, pp. 3062-3080, Dec. 2004.

[2] H. Li and Q. Zhao, "Distributed modulation for cooperative wireless communications, " IEEE Signal Processing Magazine, pp. 30-36, Sept. 2006

[3] T. Cui, F. Gao, T. Ho, and A. Nallanathan, "Distributed space-time coding for two-way wireless relay networks, "in Proc. of IEEE ICC 2008, pp. 3888-3892, 2008.

[4] C. Zhang, H. Yin, W. Wang and G. Wei, "Selective relaying schemes for distributed space-time coded relaying networks, "in IET Communications April 2010.

[5] F. A. Onat, A. Adinoyi, Y. Fan, H. Yanikomeroglu, and J. S. Thompson, "Optimum threshold for SNR-based selective digital relaying schemes in cooperative wireless networks,"in Proc. IEEE WCNC 2007, pp. 969-975, 2007.

[6] G. Scutari, S. Barbarossa, "Distributed space-time coding for regerative relay networks." IEEE Trans. Wireless Commun., vol. 4, no. 5, pp. 2387-2399, Sept. 2005.

[7] R. Hoshyar, R. Tafazolli, "A pre-BSC model for distributed turbo codes, "in Proc. of IEEE VTC 2009 Spring.

[8] P. A. Anghel, G. Leus, and M. Kaveh, "Distributed Space-time Cooperative Systems with regenerative relays, "IEEE Trans. Wireless Commun. , vol. 5, no. 11, pp. 3130-3141, Sept. 2005.

[9] F. Gao, T. Cui, and A. Nallanathan, "On channel estimation and optimal training design for Amplify and Forward relay networks, "IEEE Trans on Wireless Commun., vol. 7, no. 5, pp. 1907-1916, May. 2008.

[10] Y. Jing and B. Hassibi, "Distributed space-time coding in wireless relay networks," IEEE Trans. Wireless Commu.vol. 5, no. 12, pp. 3524-3536, Dec. 2006.

[11] S. Lin, D, J. Costello Jr., Error control coding (second edition), Pearson Education, 2004

[12] T. M. Cover, J. A. Thomas, Elements of information theory, Wiley, 1991

[13] B. Hassibi, and Haris Vikalo, "On the sphere-decoding algorithm I. Expected complexity, " IEEE Trans. Sginal Processing. vol. 53, no. 8, pp. 2806-2818, Aug. 2005

[14] H. Vikalo, B. Hassibi, and T. Kailath, "Iterative decoding for MIMO channels via modified sphere decoding," IEEE Trans. Wireless Commu.vol. 3, no. 6, pp. 2299-2311, Nov. 2004.

[15] M. N. Khormuji and E. G. Larsson, "Receiver design for wireless relay channels with regenerative relays, "in Proc. of IEEE ICC 2007.

[16] D. Chen and J. N. Laneman, "Modulation and demodulation for cooperative diversity in wireless systems," IEEE Trans. Wireless Commun., vol. 5, pp. 1785-1794, July 2006. 